\title{
Caterino, Brian: The Decline of Public Access and Neo-Liberal Media Regimes.
}

\author{
Cham: Palgrave Macmillan 2020. 280 Seiten. Preis: \\ $€ 58,84$ (e-book)
}

\section{Barbara Pfetsch}

Angenommen: 6. März 2021 / Online publiziert: 29. März 2021

(C) Der/die Autor(en) 2021

Brian Caterino bringt in seiner Studie drei Traditionslinien der Mediensoziologie zusammen: die kritische Öffentlichkeitstheorie in der Nachfolge der Frankfurter Schule, die Geschichte des öffentlichen Rundfunks und dessen Aushöhlung in den USA sowie den Aufstieg des Internets als Technologie des digitalen Kapitalismus. Das ist keine einfache Kost, zumal der Autor das große Argument wagt: Er will zeigen, dass die Bedrohung öffentlicher Medien (in den USA) nicht vom Internet ausgeht oder von anderen Technikinnovationen, sondern von einer neoliberalen Politik, welche dem Rundfunk seinen öffentlichen Auftrag abspricht. Diese These wird im Eingangskapitel des Buches klar und vermeintlich einfach formuliert. Doch was dann in den folgenden sechs Kapiteln dargelegt wird, ist eine komplexe Studie der normativen, politischen und historischen Grundlagen des öffentlichen Rundfunks in den USA. Brian Caterino argumentiert, dass die nicht-kommerziellen Medien durch den Aufstieg des Neoliberalismus existentiell gefährdet sind. Das Ergebnis ist aus seiner Sicht nicht nur das Scheitern der Idee von partizipativen demokratischen Medien, sondern auch deren Verwirklichung. Den nicht-kommerziellen Medien werde durch Gesetzgebung, Deregulierung und Profitstreben die Existenzgrundlage entzogen. Obwohl der Autor über public access und neo-liberale Medienordnungen in generalisierten Begriffen spricht, bezieht sich seine Studie ausschließlich auf die USA.

Brian Caterino demonstriert seinen LeserInnen in Kap. 2 zunächst, dass er ein hervorragender Kenner der Kritischen Theorie ist. In einer fast lehrbuchartigen Abhandlung stellt er die Ideen der Frankfurter Schule von Horkheimer bis Habermas, die Konzepte der Sozialphilosophen der modernen Zivilgesellschaft wie Cohen und Arato und die Ideen der Demokratietheorien von Barber und Alexander dem Ar-

Prof. Dr. B. Pfetsch $(\bowtie)$

Freie Universität Berlin, Garystraße 55, 14195 Berlin, Deutschland

E-Mail: pfetsch@zedat.fu-berlin.de 
gument über eine demokratische Öffentlichkeit voran, die nur durch öffentlich zugängliche Medien zu gewährleisten ist. Nach diesem theoretischen Auftakt geht es in den folgenden Kapiteln um den Aufstieg und Fall des öffentlichen Fernsehens in den USA. Die Kap. 3 und 4 sind detailreiche Ausarbeitungen der historischen Hintergründe, der gesetzlichen Grundlagen und der rechtlichen und politischen Auseinandersetzungen in der Geschichte des Public Broadcasting Service (PBS).

Während die Idee und die Institution von nicht-kommerziellen öffentlichen Rundfunkstationen in den USA bis in die 1960er-Jahre anerkannt waren, begann ihr Niedergang mit der Durchsetzung des Neoliberalismus als dominante Philosophie der politischen Ökonomie. Brian Caterino holt auch in seiner Darstellung der Grundlagen des Neoliberalismus weit aus: In Kap. 5 beschreibt er die Verbreitung der Idee und ihrer Folgen für die Kolonisierung der Lebenswelt, die Delegitimierung der Idee nicht-kommerzieller Medien, die politischen Maßnahmen der Unterminierung des PBS sowie der schleichenden Privatisierung von Öffentlichkeit. Diese Entwicklungen setzen alles in allem einen langanhaltenden Trend der Depolitisierung in Gang. Brian Caterino stellt den Wandel zunächst ideengeschichtlich und schließlich in vielen Einzelbeispielen von medienpolitischen Entscheidungen dar. Er diskutiert rechtliche Maßnahmen und politische Initiativen und zeigt, dass dem öffentlichen Rundfunk in vielen Runden der Sparpolitik die Grundlage entzogen wurde. Die Politik der Unterfinanzierung raubt dem PBS, wie Brian Caterino in Kap. 6 darlegt, die politische, inhaltliche und kulturelle Existenzberechtigung.

Das große Argument endet mit der Frage, ob das Internet die längst verschütteten Demokratieversprechen und eine emanzipatorische Öffentlichkeit wiederzubeleben vermag. Im abschließenden Kap. 7 greift Brian Caterino die aktuellen Debatten über den Wandel von Öffentlichkeit durch das Internet auf. Er setzt sich mit den Arbeiten von Castells, Keane und Fuchs auseinander und diskutiert ihre Ideen über Öffentlichkeiten in den digitalen Plattformen. Der Befund ist indessen nüchtern: Das Internet ist demnach ein durch und durch von kommerziellen Interessen dominierter Raum, der keine demokratischen Öffentlichkeiten ermöglicht. Vielmehr bestimme der Neoliberalismus auch den digitalen Kapitalismus: Manipulation und eine kommerzialisierte neoliberale Version der Konnektivität als neue Form der Ausbeutung treten an die Stelle von Dialog und Verständigung. Brian Caterino kommt am Ende seiner Studie wieder auf Habermas und die Idee einer demokratischen, verständigungsorientierten und diskursiven Öffentlichkeit zurück. Mehr Demokratie und Freiheit bewirke das Internet möglicherweise in autoritären Ländern, in demokratischen Gesellschaften dürfe man diesen Heilsbringereffekt indessen nicht erwarten.

Die Studie von Brian Caterino macht alles in allem einen zwiespältigen Eindruck. Einerseits beeindruckt der Text durch eine hervorragende Kenntnis der politischen Philosophie, der vielen Spielarten der kritischen Theorie in den USA, die in der Frankfurter Schule ihren Ausgang nimmt. Beeindruckend ist die Darstellung der politischen Ökonomie des Neoliberalismus. Hier kann der Autor aus dem Vollen schöpfen und auf seine bisherigen Arbeiten zur politischen Theorie, zur Demokratietheorie und zur Durchsetzung des neoliberalen Denkens am Ende des vergangenen Jahrhunderts zurückgreifen. Auch wenn die Fallhöhe groß ist, so erscheint die Verknüpfung dieser Ideenstränge mit einer detailreichen Recherche der Grundlagen und Entwicklung des öffentlichen Rundfunks in den USA innovativ. Andererseits über- 
zeugt die Studie nicht, denn die Rechnung geht am Ende nicht auf: die Verknüpfung der normativen demokratietheoretischen Postulate der kritischen Theorie mit der Rundfunkpolitik in den USA passen nicht wirklich zusammen.

Die Klage, dass der Neoliberalismus zur unmittelbaren Unterminierung des öffentlichen Rundfunks führt, ist nicht wirklich stringent begründet. Sie zeigt, dass der Autor das generelle Anliegen von öffentlichen nicht-kommerziellen Medienordnungen nicht verstanden hat. Es geht in der Studie nicht um neoliberale Medienordnungen an sich, sondern um eine spezielle Spielart des US-amerikanischen Mediensystems in seinen historischen und politischen Grundlagen - und wenn man genau schaut, dann hat dieses Mediensystem auch zu den besten Zeiten des National Public Radio wenig mit einer deliberativen, auf Verständigung angelegten demokratischen Öffentlichkeit gemeinsam. Brian Caterino stülpt der Krise des USamerikanischen öffentlichen Rundfunksystems einen theoretischen Überbau über, der allenfalls zeigt, dass der Autor ein exzellenter Kenner der politischen Theorie ist. Die Logiken und Spielarten öffentlicher Rundfunkorganisation und die normativen und demokratischen Implikationen dieser Form der Medienordnung hat der Autor indessen nicht wirklich überzeugend durchdrungen. Man muss daher Abstand davon nehmen, seine Argumente und Analysen auf andere real existierende Mediensysteme bzw. die europäischen Rundfunkordnungen zu übertragen.

Funding Open Access funding enabled and organized by Projekt DEAL.

Open Access Dieser Artikel wird unter der Creative Commons Namensnennung 4.0 International Lizenz veröffentlicht, welche die Nutzung, Vervielfältigung, Bearbeitung, Verbreitung und Wiedergabe in jeglichem Medium und Format erlaubt, sofern Sie den/die ursprünglichen Autor(en) und die Quelle ordnungsgemäß nennen, einen Link zur Creative Commons Lizenz beifügen und angeben, ob Änderungen vorgenommen wurden.

Die in diesem Artikel enthaltenen Bilder und sonstiges Drittmaterial unterliegen ebenfalls der genannten Creative Commons Lizenz, sofern sich aus der Abbildungslegende nichts anderes ergibt. Sofern das betreffende Material nicht unter der genannten Creative Commons Lizenz steht und die betreffende Handlung nicht nach gesetzlichen Vorschriften erlaubt ist, ist für die oben aufgeführten Weiterverwendungen des Materials die Einwilligung des jeweiligen Rechteinhabers einzuholen.

Weitere Details zur Lizenz entnehmen Sie bitte der Lizenzinformation auf http://creativecommons.org/ licenses/by/4.0/deed.de.

Prof. Dr. Barbara Pfetsch ist Professorin für Kommunikationstheorie und Medienwirkungsforschung an der Freien Universität Berlin und Principal Investigator am Weizenbaum-Institut für die Vernetzte Gesellschaft in Berlin. 